\title{
Emulating Rician distributed channels in a hybrid chamber for OTA measurements
}

\author{
Andrés Alayón Glazunov ${ }^{1,2 *}$, Oleg A. Iupikov², Pavlo S. Krasov², Robert Rehammar ${ }^{2,3}$, \\ Rob Maaskant ${ }^{2,4}$, and Marianna V. Ivashina ${ }^{2}$ \\ ${ }^{1}$ Electrical Engineering, University of Twente, Enschede, Netherlands \\ ${ }^{2}$ Electrical Engineering, Chalmers University of Technology, Gothenburg, Sweden \\ ${ }^{3}$ CTO, Bluetest AB, Gothenburg, Sweden \\ ${ }^{4}$ Electrical Engineering, Technical University Eindhoven, Eindhoven, Netherlands \\ * Corresponding author, email: a.alayonglazunov@utwente.nl
}

\begin{abstract}
It has been shown by means of simulations that the rich isotropic multipath and the random line-of-sight channels and other Rician channels in-between can be successfully generated in the same over-the-air hybrid measurement chamber without the use of additional static or moving scatterers.
\end{abstract}

Keywords-OTA, half-space RIMP, PWG, 5G and beyond.

\section{INTRODUCTION}

Generating the desired propagation channel in an Overthe-air (OTA) chamber depends on the ability of the measurement system to generate a wave field with desired statistical properties. The rich isotropic multipath (RIMP) channel can be dynamically generated in a reverberation chamber (RC) thanks, e.g., to additional moving scatterers like stirring paddles [1]. The random line-of-sight (RandomLOS) channel can be generated in semi- or anechoic chambers, where the presence of scatterers is avoided [2]. These channels are special limiting cases of the Rician channel characterized by the Rician $K$-factor parameter. Indeed, the RIMP measurement setup produces Rayleigh distributed signals with $K=0$. While in the Random-LOS measurement setup or other plane wave generator (PWG) setups a single plane wave is generated described by $K \rightarrow \infty$. Recently, a novel hybrid antenna measurement chamber has been proposed [3] consisting, in the idealized case, of an overmoded truncated waveguide with perfectly conducting walls that is terminated at its both ends with perfectly absorbing walls. The main idea is to provide further flexibility in terms of the spatial distribution of waves impinging at the device under test (DUT) by generating a single plane wave or a set of discrete plane waves incoming from pre-defined directions on the hemisphere (or half-space). The idea of generating spatial-directional channels with various Rician statistics for OTA testing of massive MIMO performance in reverberation chambers was first presented in [4]. In this paper we show that Rician channels with arbitrary $K$-factors can be successfully generated in the same hybrid OTA measurement chamber without the need of additional scatterers.

\section{Simulation SETUP}

The schematic representation of the hybrid OTA chamber model is shown in Fig. 1. The chamber has width $a=1 \mathrm{~m}$, height $b=1.25 \mathrm{~m}$ and length $c=2 \mathrm{~m}$. The side walls are assumed to be made of a perfectly conducting metal while walls at both ends are assumed to be perfectly absorbing. The chamber antenna (CHA) and the Device Under Test (DUT) are placed on opposite walls facing each other. We specialize our simulations to uniform planar arrays (UPA) for both the CHA and the DUT with inter-element spacing $d=\lambda / 2$ at the $\mathrm{CW}$ frequency of operation $f=2.5 \mathrm{GHz}$. For the CHA-UPA the elements are assumed to be vertically oriented Hertzian-dipoles, while the elements of the DUTUPA are ideal co-polarized isotropic elements. The DUTUPA has $8 \times 8$ elements, while for the CHA-UPA we consider eight array sizes, i.e., $2 n \times 2 n$, where $n=\{1,2, . .8\}$. The purpose is to study the impact of the size of the CHA-UPA on the generated field statistics.

The field at each point on the grid defined by the DUT-UPA geometry is computed employing the summation method of the waveguide (WG) modes outlined in [5]. A multiple-input multiple-output (MIMO) matrix is formed by each of the links connecting a transmit element of the CHAUPA with a receive element of the DUT-UPA. Then, various weights are applied to the input-output covariance matrix of the MIMO channel to emulate the desired statistical Rician distribution. Each entry of the $8 \times 8$ representing the

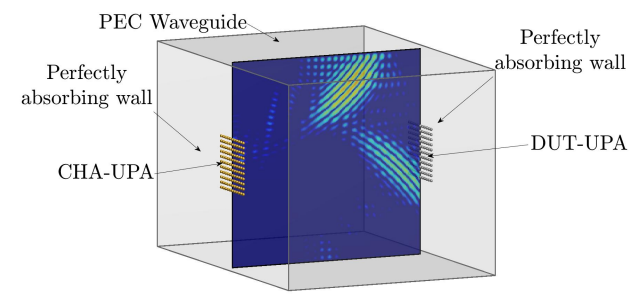

Fig. 1. Schematic representation of the hybrid OTA chamber setup. 
magnitude of the vertically polarized field is generated 1000 times. The detailed explanation of the simulation method is omitted here, but will be discussed in future publications.

\section{RESULTS AND ANALYSIS}

Fig. 2 shows a comparison of the empirical cumulative distribution functions (CDF) obtained from simulations of the vertically polarized fields in the hybrid OTA chamber and theoretical Rician CDFs denoted by solid lines and dashed lines, respectively. Different sizes of the chamber array antenna (CHA-UPA) are considered as well as different $K$-factors, $K=\{0,10,100, \infty\}$ for the emulated signals. The deterministic component propagates on-axis along the length of the waveguide while the scattered field component is confined to the hemisphere facing the CHA-UPA due to the given geometry and wave propagation within the chamber.

As can be seen from all four plots in Fig. 2, the size of the chamber array needs to be, at least, of the same size as the DUT comprising $8 \times 8$ elements. Furthermore, the statistics of the channel remain Rayleigh distributed $(K=0)$ even if the average power is erroneous when the smaller CHA-UPA are employed. However, for higher $K$-factors the shape of the $\mathrm{CDF}$ is not maintained and differs considerably from theoretical Rician CDF if the size of the chamber array is smaller than the DUT array. In order to quantify the goodness of the generated Rician distributed signals we compute the standard deviations from the empirical and the theoretical Rician CDFs. The STD for the theoretical is computed as

$$
\sigma_{\left|E_{z}\right|}=\sqrt{\left.1-\pi /(4(1+K)) L_{1 / 2}^{2}(-K)\right)}
$$

where $K$ is the Rician $K$-factor parameter and we have assumed that $\left.E\left\{\left|E_{\mathrm{z}}\right|^{2}\right\}=1 . L_{1 / 2}(x)\right)$ is the Laguerre polynomial of fractional order $1 / 2$. Results are shown in Table I, where theoretical results are provided in the rightmost column. As can be seen, a good agreement between the standard deviation computed from the empirical and the theoretical CDFs (1) is obtained for chamber arrays sizes that are equal or larger than the DUT array for the considered UPA layout. In addition we compute the root mean squared error between the empirical and theoretical CDFs as shown in Table II. Here too it's clear that the better agreement is achieved for chamber arrays equal or greater than $8 \times 8$. However, in the limiting case when a single plane wave is generated $(K \rightarrow \infty)$, the RMSE is large even if the standard deviation is practically null (see Table I). This is explained by the fact that the generated values are not uniformly distributed. This might need further investigations; however, for all practical purposes, the small standard deviation of the field amplitude will be good enough.

\section{CONCLUSION}

Rician fading channels can be generated in a hybrid overthe-air chamber without the need of additional scatterers. This is achieved by adapting the excitation coefficients of

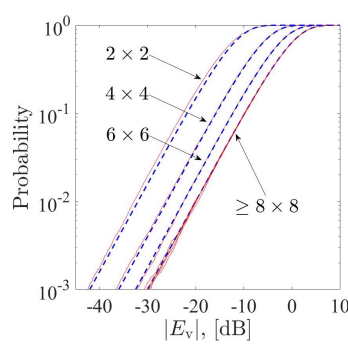

(a)

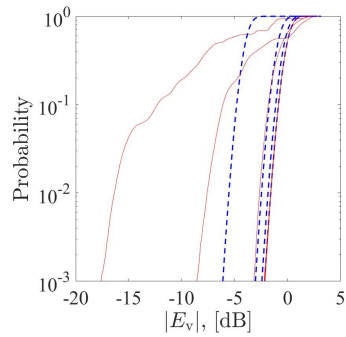

(c)

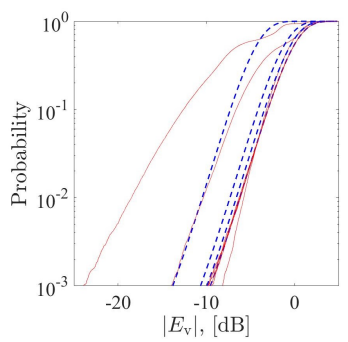

(b)

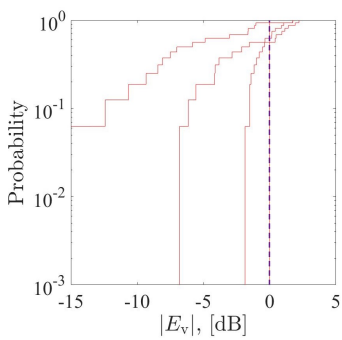

(d)
Fig. 2. Empirical cumulative distribution functions (CDF) (solid lines) and theoretical Rician CDFs (dashed lines) for different sizes of the CHA-UPA and emulated $K$-factors, (a) $K=0$, (b) $K=10$, (c) $K=100$, and (d) $K=\infty$.

TABLE I

$\operatorname{STD} \sigma_{\left|E_{\mathrm{z}}\right|}$ OF THE EMULATED RICIAN CHANNELS.

\begin{tabular}{ccccccc}
\hline \hline$K,[\mathrm{~dB}]$ & $2 \times 2$ & $4 \times 4$ & $6 \times 6$ & $8 \times 8$ & $10 \times 10$ & $\mathbf{( 1 )}$ \\
\hline$-\infty$ & 0.13 & 0.24 & 0.35 & 0.46 & 0.47 & 0.46 \\
10 & 0.27 & 0.29 & 0.19 & 0.21 & 0.21 & 0.21 \\
20 & 0.28 & 0.28 & 0.13 & 0.07 & 0.07 & 0.07 \\
$+\infty$ & 0.28 & 0.28 & 0.12 & 0.00 & 0.00 & 0.00 \\
\hline \hline
\end{tabular}

TABLE II

RMSE BETWEEN THEORETICAL AND EMULATED CDF.

\begin{tabular}{cccccc}
\hline \hline$K,[\mathrm{~dB}]$ & $2 \times 2$ & $4 \times 4$ & $6 \times 6$ & $8 \times 8$ & $10 \times 10$ \\
\hline$-\infty$ & 0.033 & 0.006 & 0.001 & 0.002 & 0.001 \\
10 & 0.208 & 0.128 & 0.009 & 0.001 & 0.001 \\
20 & 0.299 & 0.270 & 0.128 & 0.001 & 0.001 \\
$+\infty$ & 0.524 & 0.295 & 0.315 & 0.291 & 0.289 \\
\hline \hline
\end{tabular}

the chamber array such that the resulting field is proportional to the combined contribution of the half-space rich isotropic multipath and the single wave components.

\section{REFERENCES}

[1] A. Hussain, P.-S. Kildal and A. A. Glazunov, "Interpreting the Total Isotropic Sensitivity and Diversity Gain of LTE-Enabled Wireless Devices From Over-the-Air Throughput Measurements in Reverberation Chambers," IEEE Access, vol. 3, pp. 131-145, 2015

[2] M. S. Kildal, et al., "Verification of the Random Line-of-Sight Measurement Setup at 1.5-3 GHz Including MIMO Throughput Measurements of a Complete Vehicle," IEEE Trans. Veh. Technol., in press.

[3] M. Ivashina, et al., "A hybrid antenna measurement chamber," Swedish patent filed as 2030 064-6, Mar. 3, 2020.

[4] A. A. Glazunov, S. Prasad, and P. Handel, "Experimental Characterization of the Propagation Channel Along a Very Large Virtual Array in a Reverberation Chamber," Prog. Electromagn. Res. B, Vol. 59 , 205-217, 2014

[5] R. Maaskant, et al., "Analysis of a Plane Wave Generator Inside an Overmoded Rectangular Waveguide Synthesizing Oblique Incident Fields," IEEE Trans. Antennas Propagat., submitted. 